\title{
Atezolizumab in combination with Bevacizumab and Chemotherapy versus Bevacizumab and Chemotherapy in recurrent ovarian cancer - a randomized Phase III trial (AGO-OVAR 2.29 / ENGOT- ov34)
}

Philipp Harter (1), Patricia Pautier (2), Els van Nieuwenhuysen (3), Reuss A (4), Andrés Redondo (5), Kristina Lindemann (6), Christian Kurzeder (7), Edgar Petru (8), Florian Heitz (1), Jalid Sehouli (9), Nikolaus de Gregorio (10), Pauline Wimberger (11), Alexander Burges (12), Nadin Cron (13), Jonathan Ledermann (14), Domenika Lorusso (15), Xavier Paoletti (16), Frederik Marmé (17).

(1) AGO \& Ev Kliniken Essen-Mitte, Essen, Germany

(2) GINECO \& Gustave Roussy, Villejuif, France

(3) BGOG \& University Hospitals Leuven, Leuven, Belgium

(4) AGO \& KKS Marburg, Marburg, Germany

(5) GEICO \& Hospital Universitario La Paz, IdiPaz, Madrid, Spain

(6) NSGO \& Oslo University Hospital, Unversity of Oslo, Oslo, Norway

(7) SAKK \& University Hospital of Basel, Basel, Switzerland

(8) AGO-Austria \& Graz University, Graz, Austria

(9) AGO \& Charité Berlin, Berlin, Germany

(10) AGO \& University Ulm, Ulm, Germany

(11) AGO \& TU Dresden, Dresden, Germany

(12) AGO \& LMU Munich, Munich, Germany

(13) AGO, Essen, Germany

(14) UCL Cancer Institute, University College, London, UK

(15) Policlinico Gemelli, Rome, Italy

(16) Institute Curie, Paris, France

(17) AGO \& University Mannheim, Mannheim, Germany

\section{Corresponding author:}

Dr. Philipp Harter

Department of Gynecology \& Gynecologic Oncology

Ev. Kliniken Essen-Mitte

Henricistrasse 92

45136 Essen

Germany 
Phone: +4920117434511

e-mail: p.harter@gmx.de

\begin{abstract}
:
Background: Improvement of clinical outcomes of patients with platinum resistant disease is an unmet medical need and trials in this population are urgently needed. Checkpointinhibitors have already shown activity in multiple other tumor entities and ovarian cancer, especially in the combination with anti-angiogenic treatment.
\end{abstract}

Primary Objective: To test, if the activity of non-platinum-based chemotherapy and bevacizumab could be improved by the addition of atezolizumab.

Study Hypothesis: The addition of atezolizumab to standard non-platinum combination of chemotherapy and bevacizumab improves median overall survival from 15 to 20 months.

Trial Design: Patients are randomized to chemotherapy (paclitaxel weekly or pegylated liposomal doxorubicin) + bevacizumab + placebo versus chemotherapy + bevacizumab + atezolizumab. Stratification factors are: Number of prior lines, planned type of chemotherapy, prior use of bevacizumab, and tumor PD-L1 status.

Major Inclusion/Exclusion Criteria: Recurrent epithelial ovarian, fallopian tube, or primary peritoneal cancer with up to three prior therapies. In patients with 1 or 2 prior treatment lines, the treatment free interval after platinum has to be less than 6 months.Patients with three prior lines of chemotherapy who are not considered for platinum-containing chemotherapy lines are also eligible. A de novo tumor tissue sample biopsy for determination of PD-L1 status prior to randomization for stratification is mandatory. Major exclusion criteria consider bevacizumab specific and immunotherapy specific criteria.

Primary Endpoint: Overall survival and progression free survival are co-primary endpoints

Sample Size: It is planned to randomize 664 patients

Trial Registration: NCT03353831

Key words: ovarian cancer, relapse, platinum resistant disease, bevacizumab, atezolizumab, phase III trial

\title{
Background:
}

Despite optimal primary surgery and carboplatin/paclitaxel based chemotherapy, the majority of patients with newly diagnosed advanced ovarian cancer will relapse. Treatment options for recurrent disease depend on treatment free interval, prior therapy, BRCA-status, symptoms and patient's preference. Further treatment depends on eligibility for further platinum-based treatment. In patients who are not eligible for another platinum-based treatment, a nonplatinum-based drug like pegylated liposomal doxorubicin (PLD), gemcitabine, paclitaxel, or topotecan is the cytotoxic treatment of choice. The AURELIA trial showed a significant PFS benefit of adding bevacizumab to single agent chemotherapy in this patient cohort (HR 0.48; $95 \% \mathrm{Cl} 0.38-0.60 ; p<0.001)\left[{ }^{1}\right]$. Therefore, bevacizumab based treatment is a standard option in patients with platinum-resistant ovarian cancer. 
A spontaneous antitumor immune response has been demonstrated in approximately $55 \%$ of the patients with $\mathrm{OC}$ in the form of tumor-infiltrating lymphocytes (TILs) [ $\left.{ }^{2}\right]$ which has been repeatedly associated with a prolonged survival among ovarian cancer patients [ $\left.{ }^{3}\right]$. Pembrolizumab, an anti-PD1 humanized antibody achieved 3 confirmed responses $(11.5 \%$ [ $(95 \% \mathrm{Cl}, 2.4-30.2])$ in 26 patients treated in a phase IB study and 3 additional patients had a tumor reduction of at least $30 \%\left[{ }^{4}\right]$. In a small study including 12 patients who received $\geq 2$ previous therapy lines in advanced ovarian cancer, at increasing doses of $0.3-15 \mathrm{mg} / \mathrm{kg}$, safety, clinical activity and biomarkers of atezolizumab have been studied. Treatment-related AEs were mainly grade I/II fatigue and pain. Two out of 9 evaluable patients at the 10 or 15 $\mathrm{mg} / \mathrm{kg}$ dose levels showed a long lasting response [5].

There is increasing evidence that VEGF plays a role in cancer immune evasion through several different mechanisms [ $\left.{ }^{6}\right]$. A randomized phase III trial with 915 patients in metastatic renal cell carcinoma has shown superiority of bevacizumab in combination with atezolizumab versus sunitinib. Safety was comparable to the known individual profiles of atezolizumab and bevacizumab $\left.{ }^{7}\right]$. Further supporting evidence for the combination of atezolizumab and bevacizumab has been shown in IMpower 150 in non-small-cell lung cancer [ ${ }^{8}$ ].

Because of the intimate relationship between angiogenesis and immunosuppression, it is expected that inhibiting both pathways will result in improved and more durable clinical benefit.

Therefore, we aim to test the efficacy of atezolizumab in combination with non-platinum-based chemotherapy and bevacizumab versus the combination of a non-platinum-based chemotherapy and bevacizumab alone.

After initiation of study recruitment in Germany, data from a phase III trial in platinum-resistant ovarian cancer, JAVELIN $\left.200{ }^{9}\right]$, with avelumab, a monoclonal anti-PD-L1 antibody was reported and suggested that PD-L1 positive patients derived a greater benefit than PD-L1 negative patients. To date there is no accepted gold standard for immuno-histochemistry for ovarian cancer based PD-L1 testing and diagnostic assays, scoring methods as well as cutoffs vary between different tumor types and therapeutic antibodies. However, overall there are consistent reports from several tumor entities, that PD-L1 status is a predictive factor for benefit from immune-checkpoint inhibitors. Based on the available data from atezolizumab in ovarian cancer and other solid tumors, we therefore implemented PD-L1 testing using the VENTANA SP142 diagnostic assay as a stratification factor and in addition, we will explore alternative PD-L1 testing assays, aiming to best define the population, which will benefit the most from immune-checkpoint inhibitors. This major amendment was approved by the authorities in January 2020 after recruitment of 94 patients.

\section{Methods}

This is an international placebo-controlled randomized phase III trial, which is conducted as an European Network of Gynaecological Oncological Trial Groups (ENGOT) trial according to model $\left.A{ }^{10}\right]$. The target population includes patients with recurrent ovarian-, fallopian tube, or primary peritoneal carcinoma with 1st or 2nd relapse within 6 months after platinum-based chemotherapy or 3rd relapse. Eligible patients undergo a biopsy for testing PD-L1 status. After obtaining the result, patients are randomized to chemotherapy + bevacizumab + placebo (standard arm) versus chemotherapy + bevacizumab + atezolizumab. Allowed chemotherapy regimen include paclitaxel and PLD according to prior treatment and investigators decision. The allowed chemotherapy backbone is weekly paclitaxel $80 \mathrm{mg} / \mathrm{m}^{2}$ and PLD $40 \mathrm{mg} / \mathrm{m}^{2} \mathrm{~d} 1 \mathrm{q} 28$. Bevacizumab is dosed at $10 \mathrm{mg} / \mathrm{KG} \mathrm{d1,15} \mathrm{q} 28$ and atezolizumab/placebo at $840 \mathrm{mg}$ d1,15,q28. Stratification factors are: Number of prior treatment lines (1-2 vs. 3), planned 
chemotherapy (PLD vs. paclitaxel), previous administration of bevacizumab (yes vs. no), and tumor PD-L1 status (figure 1).

\section{Participants:}

Major inclusion criteria are patients with histologically diagnosed epithelial ovarian, fallopian tube, or primary peritoneal cancer and relapsed disease. Up to three prior therapies are allowed, in patients with 1 or 2 prior treatment lines, the treatment free interval after platinum has to be less than 6 months; in addition patients with three prior lines of chemotherapy who are not considered for platinum-containing chemotherapy lines are also eligible. Patients must have measurable disease, evaluable disease in combination with GCIG CA-125 criteria, or histologically proven re-lapse/progression. In addition, a de novo tumor biopsy (not older than 3 months) sent to central laboratory as formalin-fixed, paraffin-embedded (FFPE) sample for determination of PD-L1 status prior to randomization for stratification is mandatory. Major exclusion criteria consider bevacizumab specific and immunotherapy specific criteria. Of note, prior use of bevacizumab is allowed.

\section{Outcomes}

The primary objective of this study is to determine the efficacy of atezolizumab plus bevacizumab and chemotherapy compared with placebo plus bevacizumab and chemotherapy in patients with recurrent ovarian-, fallopian tube, or primary peritoneal cancer with 1st or 2nd relapse within 6 months after platinum-based chemotherapy or 3rd relapse. There are 2 coprimary endpoints:

- Overall survival (OS) defined as the time from randomization to death from any cause

- Progression free survival (PFS) defined as the time from randomization to progressive disease (PD) or death, whichever occurs earlier. PD is based on investigator assessment using the Response Evaluation Criteria in Solid Tumors (RECIST 1.1).

Secondary endpoints include Quality of Life and patient reported outcomes (PRO) measured by EORTC QLQ-C-30, QLQ-OV28, PRO-CTCAE in the whole study population and in subgroups. Further secondary endpoints include response rate, safety, tolerability and efficacy in biomarker defined subgroups.

To monitor patient safety and supervise the progress of the trial an independent data monitoring committee has been established. The first IDMC meeting took place after 24 patients had been randomized and had completed at least cycle 1. There were no concerns to continue without any changes.

\section{Randomization and Blinding}

The randomization procedure differed between the first 24 patients (run-in phase) and the rest of the patients. These first 24 patients were allocated to arm $A$ and arm $B$ in a 1:1 ratio balancing for the stratification factor planned chemotherapy (12 patients with paclitaxel and 12 patients with PLD). The recruitment was interrupted for the safety analysis.

Random assignment for all other patients follows a 1:1 ratio to the treatment arms with the following stratification factors:

- Number of prior lines (1-2 vs. 3)

- Planned chemotherapy (PLD vs. paclitaxel) 
- Previous administration of bevacizumab (yes vs. no)

- Tumor PD-L1 status (positive vs negative vs non-informative). The inclusion of patients with non-informative tissue PD-L1 status will be capped to $10 \%$ of the whole study population (this additional strata was implemented by the above mentioned amendment). Randomization lists with randomly permuted block sizes were prepared by KKS, Philipps-University of Marburg, Germany. Randomization is performed by the IWRS-system of S-CLINICA.

\section{Sample size calculation}

We assume a median OS of 15 months in the control arm and 20 months in the experimental arm (i.e. hazard ratio of 0.75 ) and a median PFS of 6.5 months resp. 9.3 months (hazard ratio of 0.70 ). Further assumptions are a 24 months accrual phase followed by approximately 13.2 months follow-up, exponential distributions of OS and PFS and a drop-out rate of $5 \%$. The analysis is planned with a power of $80 \%$ for the OS comparison. The sample size and power calculations were performed with ADDPLAN (v.6.1.0).

\section{Statistical Methods}

Each of the co-primary endpoints OS and PFS will be compared according to the intention to treat principle using the Wald-test for the treatment variable from a Cox model with the treatment arm and randomization stratification factors as covariables. The two-sided significance levels are 0.005 for PFS and 0.045 for OS. Secondary endpoints will be analyzed with appropriate statistical methods.

\section{Discussion}

Treatment of patients in whom a platinum-based chemotherapy is no longer a good treatment option is still one of the most challenging treatment situations. Usual single agent chemotherapies like paclitaxel, PLD, topotecan and gemcitabine show very limited activity. Therefore, best supportive care without any further tumor-directed therapy is also a standard option. So far, one of the only positive phase III trials in the last years was the AURELIA trial which showed superiority by the addition of bevacizumab to standard chemotherapy regarding PFS and Quality of Life. Therefore, single agent non-platinum based chemotherapy in combination with bevacizumab is a new standard. Unfortunately, this regimen is only approved by authorities in the European Union in patients without prior use of bevacizumab. The use of bevacizumab already in the first or second line setting $\left[{ }^{11},{ }^{12}\right]$ limits it use in the resistant population due to this regulatory restrictions. However, there is growing evidence regarding retreattment with bevacizumab in colorectal and ovarian cancer $\left[{ }^{13},{ }^{14}\right]$. As we allowed prior use of bevacizumab in this trial, we will also be able to provide data for the re-usage of bevacizumab in combination with non-platinum agents.

So far there is no phase II data regarding safety of this combination in our patient population which is characterized by more fragility, tumor symptoms and persisting toxicities compared to patients in earlier lines of treatment. We therefore had to focus on safety issues and started a safety run-in phase with 24 patients who completed at least cycle 1 and we stopped the trial before further patients could be randomized. Safety data were monitored by IDMC, which had no concerns. Next IDMC meetings are planned after additional 60 and 120 randomized patients who completed at least cycle 1 (without stopping the trial).

Important for the conduct of this trial was the presentation of the JAVELIN 200 trial in 2019. In Javelin 200 a similar patient population was randomized in a 3-arm trial to PLD, avelumab or the combination. The trial did not meet its primary endpoint but a subgroup analysis suggested that a benefit by the addition of an immunotherapy is limited to patients with a positive PD-L1 status. Unfortunately, the full publication of JAVELIN 200 and further data regarding the predictive role of the PD-L1 status in prospective randomized trials in ovarian cancer is still 
missing. Considering this new evidence, we decided to add PD-L1 status as stratification factor in our trial. In addition, it is unclear in ovarian cancer, if the PD-L1 status in the primary tumor compared to metastases, over time and multiple treatment lines remains stable. Therefore, we decided to perform PD-L1 staining on fresh biopsies to rule out uncertainty of the recent PDL1 status before inclusion in the trial.

In conclusion, AGO-OVAR 2.29/ENGOT-ov 34 is the logical further development of the so far most efficacious regimen in platinum resistant ovarian cancer. Because the primary endpoint includes overall survival as recommended by the $5^{\text {th }}$ Ovarian Cancer Conference $\left[{ }^{15}\right]$, the trial will be able to provide a definitive answer of this new treatment option in ovarian cancer patients.

\section{Acknowledgements}

We would like to thank the participating study groups AGO Austria, BGOG, GEICO, GINECO, NSGO, and SAKK. In addition, we would like to thank F. Hoffmann-La Roche Ltd for financial support and providing the study drugs. Furthermore, we would like to thank lqbal Hügel and his team from SPS for monitoring services.

\section{Figures and tables}

Figure 1) Study design of AGO-OVAR 2.29/ENGOT-ov 34

\footnotetext{
${ }^{1}$ Pujade-Lauraine E, Hilpert F, Weber B, et al. Bevacizumab combined with chemotherapy for platinumresistant recurrent ovarian cancer: The AURELIA open-label randomized phase III trial. J Clin Oncol. 2014; 32:1302-08.

${ }^{2}$ Zhang L, Conejo-Garcia JR, Katsaros D, et al. Intratumoral T cells, recurrence, and survival in epithelial ovarian cancer. N Engl J Med. 2003; 348(3):203-13.

${ }^{3}$ Hwang WT, Adams SF, Tahirovic E, et al. Prognostic significance of tumor-infiltrating T cells in ovarian cancer: a meta-analysis. Gynecol Oncol. 2012; 124(2):192-8.

${ }^{4}$ Varga A, Piha-Paul SA, Ott PA, et al. Pembrolizumab in patients with programmed death ligand 1-positive advanced ovarian cancer: Analysis of KEYNOTE-028. Gynecol Oncol. 2019 Feb;152(2):243-250

${ }^{5}$ Liu JF, Gordon M, Veneris J, et al. Safety, clinical activity and biomarker assessments of atezolizumab from a Phase I study in advanced/recurrent ovarian and uterine cancers. Gynecol Oncol. 2019 Aug;154(2):314-322 ${ }^{6} \mathrm{Ohm}$ JE, Carbone DP. VEGF as a Mediator of Tumor-Associated Immunodeficiency. Immunology Research 2001; 23-2/3:263-72.

${ }^{7}$ Rini BI, Powles T, Atkins MB, et al. Atezolizumab plus bevacizumab versus sunitinib in patients with previously untreated metastatic renal cell carcinoma (IMmotion151): a multicentre, open-label, phase 3, randomised controlled trial. Lancet. 2019 Jun 15;393(10189):2404-2415.

${ }^{8}$ Socinski MA, Jotte RM, Cappuzzo F, et al. Atezolizumab for First-Line Treatment of Metastatic Nonsquamous NSCLC. N Engl J Med. 2018 Jun 14;378(24):2288-2301.

${ }^{9}$ Pujade-Lauraine E, Fuijwara K, Lederman JA et al. Avelumab alone or in combination with pegylated liposomal doxorubicin vs. pegylated liposomal alone in platinum-resistant or refractory epithelial ovarina cancer: primary and biomarker analysis of the phase 3 JAVELIN Ovarian 200 trial. Society of Gynecologic Oncology. 2019, Vol. 50th Annual Meeting.

${ }^{10}$ du Bois A, Reuss A, Pujade-Lauraine E, et al. European Network of Gynaecological Oncological Trial Groups' Requirements for Trials Between Academic Groups and Industry Partners--First Update 2015. Int J Gynecol Cancer. 2015 Sep;25(7):1328-30.

${ }^{11}$ Harter P, Pfisterer J, Hilpert F, et al. Therapiequalität des fortgeschrittenen Ovarialkarzinoms in Deutschland. Frauenarzt 2020; 61(3): 182-188

12 Pfisterer J, Shannon CM, Baumann K, et al. Bevacizumab and platinum-based combinations for recurrent ovarian cancer: a randomised, phase 3 trial. Lancet Oncol April 16, 2020; https://doi.org/10.1016/S14702045(20)30142-X
} 
${ }^{13}$ Masi G, Salvatore L, Boni L, et al. Continuation or reintroduction of bevacizumab beyond progression to firstline therapy in metastatic colorectal cancer: final results of the randomized BEBYP trial. Ann Oncol. 2015 Apr;26(4):724-30.

${ }^{14}$ Pignata S, Lorusso D, Joly F, et al. Chemotherapy plus or minus bevacizumab for platinum-sensitive ovarian cancer patients recurring after a bevacizumab containing first line treatment: The randomized phase 3 trial MITO16B - MaNGO OV2B - ENGOT OV17. J Clin Oncol 2018; 36 (suppl; abstr 5506)

${ }^{15}$ Bookman MA, Okamoto A, Stuart G, et al. Harmonising clinical trials within the Gynecologic Cancer InterGroup: consensus and unmet needs from the Fifth Ovarian Cancer Consensus Conference. Ann Oncol. 2017 Nov 1;28(suppl_8):viii30-viii35

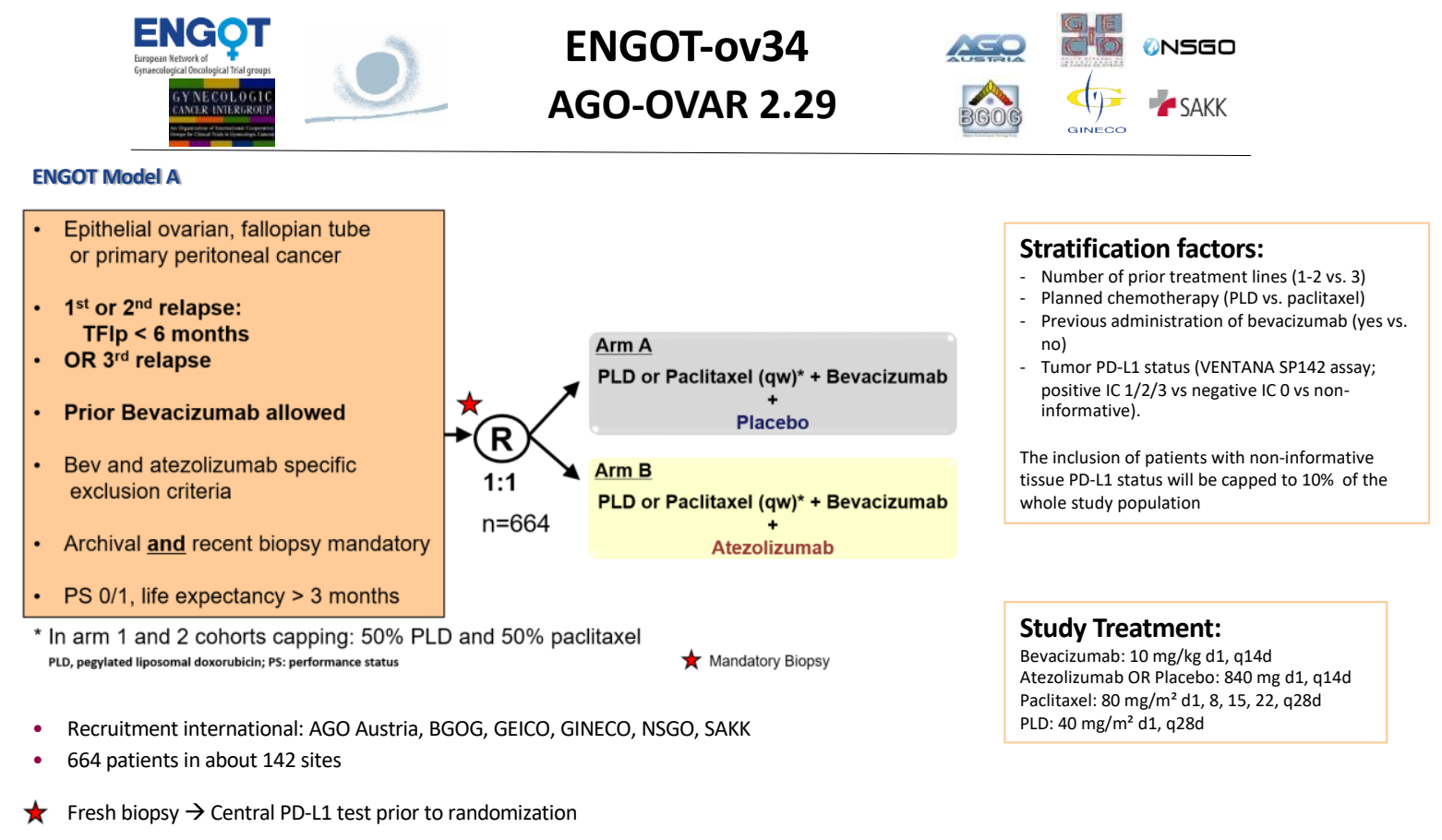

